\title{
Jejunal Fistula, CTCAE
}

National Cancer Institute

\section{Source}

National Cancer Institute. Lejunal Fistula, CT CAE. NCI Thesaurus. Code C57827.

A disorder characterized by an abnormal communication between the jejunum and another organ or anatomic site. 